\title{
ECDC publishes 2013 surveillance data on antimicrobial resistance and antimicrobial consumption in Europe
}

K Weist (klaus.weist@ecdc.europa.eu) $)^{1,2}$, L Diaz Högberg ${ }^{1,2}$

1. Surveillance and Response Support Unit, European Centre for Disease Prevention and Control (ECDC), Stockholm, Sweden

2. The European Centre for Disease Prevention and Control (ECDC) Antimicrobial Resistance and Healthcare-Associated Infections Programme

Citation style for this article:

Weist K, Diaz Högberg L. ECDC publishes 2013 surveillance data on antimicrobial resistance and antimicrobial consumption in Europe . Euro Surveill. 2014;19(46): pii=20962. Available online: http://www.eurosurveillance.org/ViewArticle. aspx?Articleld=20962

Article published on 20 November 2014

On the occasion of the European Antibiotic Awareness Day (EEAD) on 18 November 2014, the European Centre for Disease Prevention and Control (ECDC) has released 2013 data on antimicrobial resistance and antimicrobial consumption in Europe.

The ECDC collects data on antimicrobial resistance and antimicrobial consumption from 30 European Union (EU) and European Economic Area (EAA) countries through the European Antimicrobial Resistance Surveillance Network (EARS-Net) and the European Surveillance of Antimicrobial Consumption Network (ESAC-Net). Surveillance data for 2013 are provided through openly accessible online interactive databases $[1,2]$, in summaries supporting the EAAD event [3] and, for EARS-Net, in the annual report on antimicrobial resistance surveillance in Europe [4].

EARS-Net data for 2013 confirmed the high percentages of invasive isolates of Klebsiella pneumoniae and Escherichia coli with resistance to third-generation cephalosporins that were reported in the previous three years. For $K$. pneumoniae, EARS-Net observed an increase, during the period $2010 \square 2013$, of the EU/ EEA population-weighted mean percentage of isolates showing combined resistance to three major classes of antibiotics and of isolates with resistance to carbapenems.

ESAC-Net data for 2013 showed that overall consumption of antibiotics (ATC group J01, antibacterials for systemic use) in the hospital sector remained stable in the EU/EEA. However, the EU/EEA population-weighted mean consumption of carbapenems and of polymyxins both increased during $2009 \square 2013$. This increase may possibly reflect overuse, or may simply indicate an increased clinical need for these antibiotics in hospital settings because of an increasing prevalence of infections with multidrug-resistant Gram-negative bacteria in Europe.
For the first time, EARS-Net reported data on polymyxin resistance, showing the presence of such resistance in all Gram-negative bacteria under surveillance, especially in countries with already high levels of carbapenem resistance. This new, worrisome development means that, for patients with serious infections with such carbapenem- and polymyxin-resistant Gramnegative pathogens, only few alternatives remain available for appropriate antimicrobial treatment.

Antimicrobial resistance, especially to last-line antibiotics, is a serious threat to public health and patient safety in Europe. It would be worthwhile to link antimicrobial consumption data from ESAC-Net with antimicrobial resistance data from EARS-Net in order to gain a better understanding of the dynamic relationship between these parameters in the hospital sector in Europe. A prerequisite for such an integrated analysis would require a hospital-based reporting of antimicrobial consumption to ESAC-Net as well as use of a unique, though confidential, hospital identifier for reporting data to both EARS-Net and ESAC-Net.

\section{References}

1. European Centre for Disease Prevention and Control (ECDC). Antimicrobial resistance interactive database (EARS-Net). Available from: http://www.ecdc.europa.eu/en/healthtopics/ antimicrobial_resistance/database/Pages/database.aspx

2. European Centre for Disease Prevention and Control (ECDC). Antimicrobial consumption interactive database (ESAC-Net). Available from: http://www.ecdc.europa.eu/en/healthtopics/ antimicrobial_resistance/esac-net-database/Pages/database. aspx

3. European Centre for Disease Prevention and Control (ECDC). Summaries of the latest data on antimicrobial resistance and antimicrobial consumption in EU: 2014. Available from: http:// ecdc.europa.eu/en/eaad/Pages/antibiotics-data-reports.aspx

4. European Centre for Disease Prevention and Control (ECDC). Antimicrobial resistance surveillance in Europe 2013. Annual Report of the European Antimicrobial Resistance Surveillance Network (EARS-Net). Stockholm: ECDC; 2014. Available from: http://www.ecdc.europa.eu/ en/publications/_layouts/forms/Publication_DispForm. aspx? List $=4 \mathrm{f}_{55}$ ad $51-4$ aed-4d32-b960-af70113dbb90\&ID = 1205 